\title{
Periorbital Edema, CTCAE
}

National Cancer Institute

\section{Source}

National Cancer Institute. Periorbital Edema, CT CAE. NCI Thesaurus. Code C143747.

A disorder characterized by swelling due to an excessive accumulation of fluid around the orbits of the face. 